\title{
Research Article \\ Study of High Efficiency Flow Regulation of VIGV in Centrifugal Compressor
}

\author{
Chunjun Ji, ${ }^{1,2}$ Qi Sun, ${ }^{1}$ Zhaoyang Fan, ${ }^{1}$ Yawei Gao, ${ }^{1}$ and Baode Zhao \\ ${ }^{1}$ School of Energy and Power Engineering, Dalian University of Technology, Dalian 116024, China \\ ${ }^{2}$ Key Laboratory of Ocean Energy Utilization and Energy Conservation of Ministry of Education, \\ Dalian University of Technology, Dalian 116024, China
}

Correspondence should be addressed to Qi Sun; sunqi525@126.com

Received 24 November 2015; Revised 2 February 2016; Accepted 3 February 2016

Academic Editor: Ryoichi Samuel Amano

Copyright (C) 2016 Chunjun Ji et al. This is an open access article distributed under the Creative Commons Attribution License, which permits unrestricted use, distribution, and reproduction in any medium, provided the original work is properly cited.

\begin{abstract}
Variable inlet guide vane (VIGV) is used to control the mass flow and generate prewhirl in centrifugal compressors. Due to the tip clearance of the guide vanes and the defect of the traditional guide vane profiles, the mass flow regulation of VIGV is limited, resulting in a large waste of compressed gas. Two kinds of inlet flow channels were proposed to eliminate the influence of tip clearance. These structures were numerically investigated at different setting angles. The results show that the improved channels not only expand the range of mass flow regulation, but also reduce the power and increase the efficiency of the compressor. Ten kinds of guide vane profiles, including different thickness distribution, camber line profile, were selected to compare with the original one and with each other. In the premise of ensuring the performance of compressor, the best guide vane profile was selected. The results show that reducing the guide vane thickness, increasing the guide vane camber angle, and increasing the distance between the maximum camber position and the leading edge of guide vane can help expand the range of mass flow regulation. The achievement of this research can effectively improve the flow regulation ability of VIGV and the performance of compressor.
\end{abstract}

\section{Introduction}

In order to adapt to climate change and to meet the user requirements, the gas mass flow in actual operation of a centrifugal compressor needs to be regulated normally [1]. Variable inlet guide vane (VIGV) in the first stage of an centrifugal compressor is one of the effective ways to realize this function [2]. However, the regulating ability of traditional VIGV is very limited, resulting in a large amount of compressed gas that has to be discharged without effective use because of overproduction, especially in winter, up to $30 \%$ in some cases [3]. So it is very important to develop high efficiency VIGV device to solve the problem.

The purpose of the flow regulation is achieved with the flow area change and prewhirl generation of inlet gas by turning the VIGV angle in the operation of the centrifugal compressor [4]. However, a part of gas flow near the inlet channel wall cannot be controlled because of the tip clearance when the VIGV turns into a certain angle, especially at the large setting angle [5].
As the guide vane rotating to a certain angle, in order to avoid the collision between the guide vane and the casing wall, there must be a tip clearance on the top of inlet guide vane. Therefore, it is necessary to make the top of guide vane into a circular arc shape to match the casing wall when it turns an angle. When the fluid flows through the inlet guide vane passage, the pressure between the suction side and the pressure side of guide vane is different. It will lead to a portion of gas to climb over the tip clearance on the top of guide vane and then mix with the mainstream. This part of the gas cannot be regulated by the guide vanes, and the mix may cause some extra flow losses.

In addition, the shape of the VIGV is also a key factor to affect the flow regulation. Most VIGVs have symmetric vane profiles and create similar inlet flow characteristics at positive and negative prewhirls. At nonzero setting angles, flow experiences a strong suction side acceleration in the vicinity of the leading edge. The subsequent large deceleration can cause massive flow separation already at low incidence, which limits the useful operating range of VIGV [6]. In 


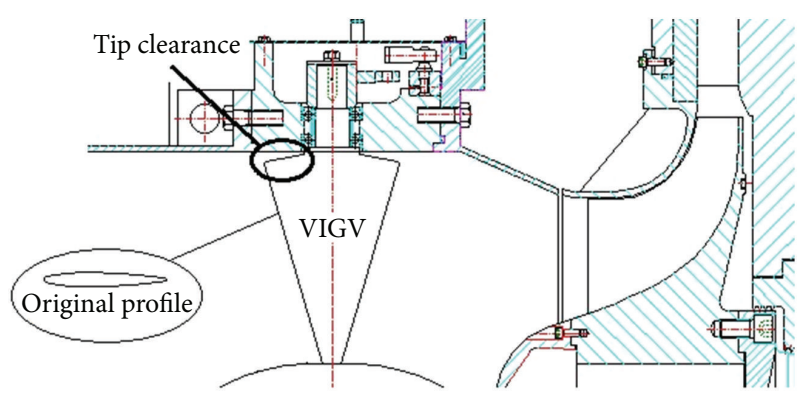

FIGURE 1: Sketch of VIGV in centrifugal compressor.

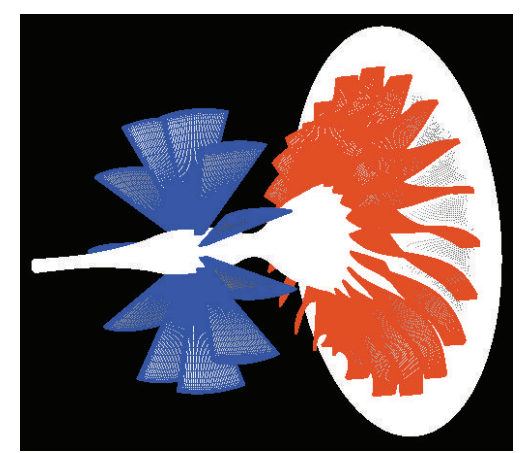

FIGURE 2: Mesh division of original model.

order to solve these problems and improve the regulation performance of VIGV, the thickness and camber and the position of maximum camber of guide vane were studied to find the most suitable profile, thereby expanding the flow regulation range of VIGV and improving the performance of the compressor.

The aim of this paper is to eliminate the influence of the tip clearance and select the best guide vane profile to achieve the optimal flow regulation [7].

In this paper, a centrifugal compressor (the first stage) in operation of one factory was provided as the research object. In the operation of this compressor, a large amount of compressed gas was often wasted due to the limitation of the regulating capacity of the inlet guide vanes. The compressor is composed of an inlet chamber, a VIGV system of 11 symmetrical straight vanes, an impeller of 19 rear curved semi-open impeller blades, and a diffuser. Figure 1 shows the sketch of this compressor.

\section{Numerical Calculation Method}

The model of the compressor, including the VIGV system and the impeller, was developed by the Pro/E software. High quality structural grid was created by NUMECA Autogrid5 [8]. The structure of grid is $\mathrm{H}-\mathrm{I}$, and the total number is about 16 million in the whole flow passage. In this paper, the same mesh topology and the number of grids were used for all the computational model. Figure 2 shows the mesh division of original model.

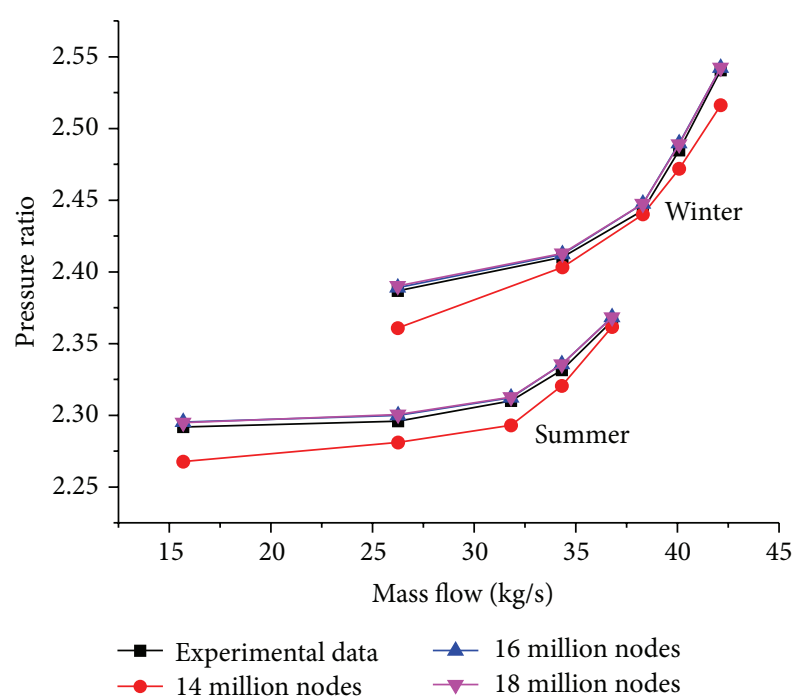

FIGURE 3: Comparison of pressure ratio curve in different number of grid nodes and experimental data.

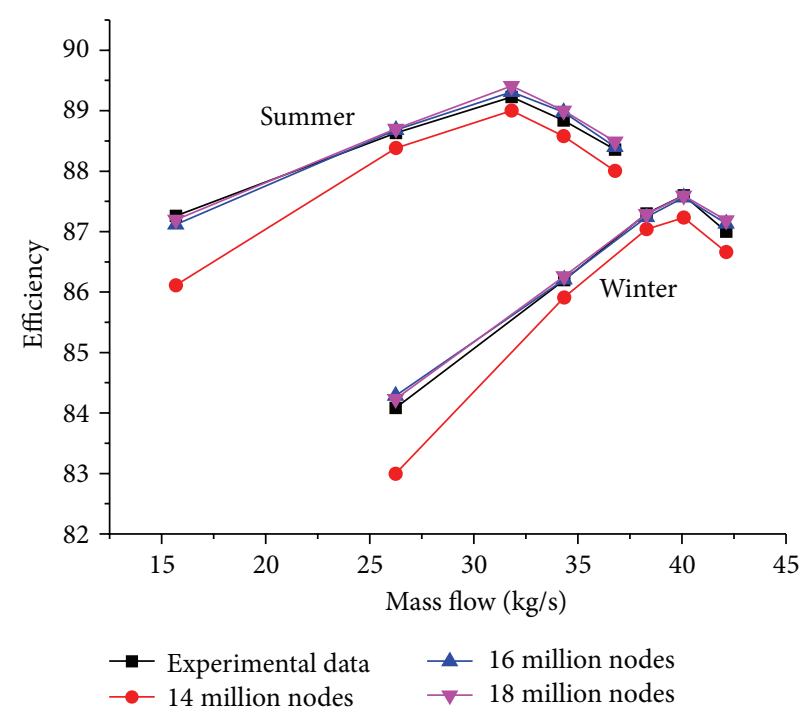

FIGURE 4: Comparison of efficiency curve in different number of grid nodes and experimental data.

In order to verify the accuracy of numerical calculation, three different numbers of grid nodes (14 million nodes, 16 million nodes, and 18 million nodes) and the original experimental data in the real operation process of the compressor provided from the factory were used to compare as shown in Figures 3 and 4. The comparison result shows that the numbers of 16 million nodes and 18 million nodes agree quite well with the original experimental data. It is clear that 16 million nodes can meet the requirement of calculation and the numerical method used in this paper is reliable.

The performance at different guide vane setting angles $\left(-20^{\circ}, 0^{\circ}, 20^{\circ}, 40^{\circ}, 60^{\circ}\right)$ in summer and winter was analyzed by means of NUMECA Fine [8]. The time-averaged NavierStokes equations were solved by time marching method, with the Spalart-Allmaras turbulence model. The $y+$ was limited 
TABLE 1: Calculation parameters and boundary conditions.

\begin{tabular}{lc}
\hline Item & Setting \\
\hline Working medium & Air \\
Impeller speed & $7460 \mathrm{r} / \mathrm{min}$ \\
Design point mass flow & $32.29 \mathrm{~kg} / \mathrm{s}$ \\
Inlet total temperature & $303 \mathrm{~K} \mathrm{in} \mathrm{summer}$ \\
Inlet total pressure & $263 \mathrm{~K} \mathrm{in} \mathrm{winter}$ \\
Turbulent viscosity & $97000 \mathrm{~Pa}$ \\
Inlet condition & $5 \times 10^{-5} \mathrm{~m}^{2} / \mathrm{s}$ \\
Outlet condition & Axial inlet flow \\
\hline
\end{tabular}

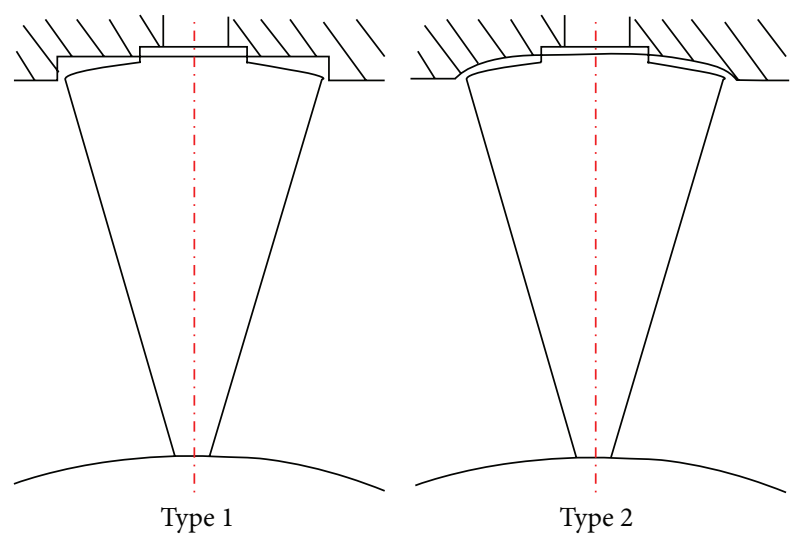

FIgURE 5: Two kinds of improved inlet flow channels.

to 10 as the requirement of low Reynolds number turbulence model according to the recommendation of NUMECA. The convergence of the algorithm was accelerated by using the full multigrid and implicit residual smoothing technique. The calculation parameters and boundary conditions were shown in Table 1.

\section{Modification of Inlet Flow Channel}

In order to eliminate the influence of the tip clearance of the guide vanes on the performance of the flow regulation, two kinds of inlet flow channels were proposed, as shown in Figure 5. Type 1 is to wrap the tip clearance in a right angle annular channel. Type 2 is to wrap the tip clearance by the circular arc annular channel. In order to verify the validity of these two improved channels, the same numerical calculation method was used to analyze and compare the performance of the two modified channels with the original structure.

From Figure 6, we can see that the two improved channels can reduce the flow rate at the large setting angle, so that it can help to expand the range of mass flow regulation. The adjustment range of Type 2 is significantly higher than that of Type 1 . The regulation range of Type 2 is expanded by $15.19 \%$ in summer and by $9.16 \%$ in winter.

As can be seen from Figure 7, the improved channels have no effect on the power of the compressor at low setting angle of VIGV, but the power can be reduced with the increase

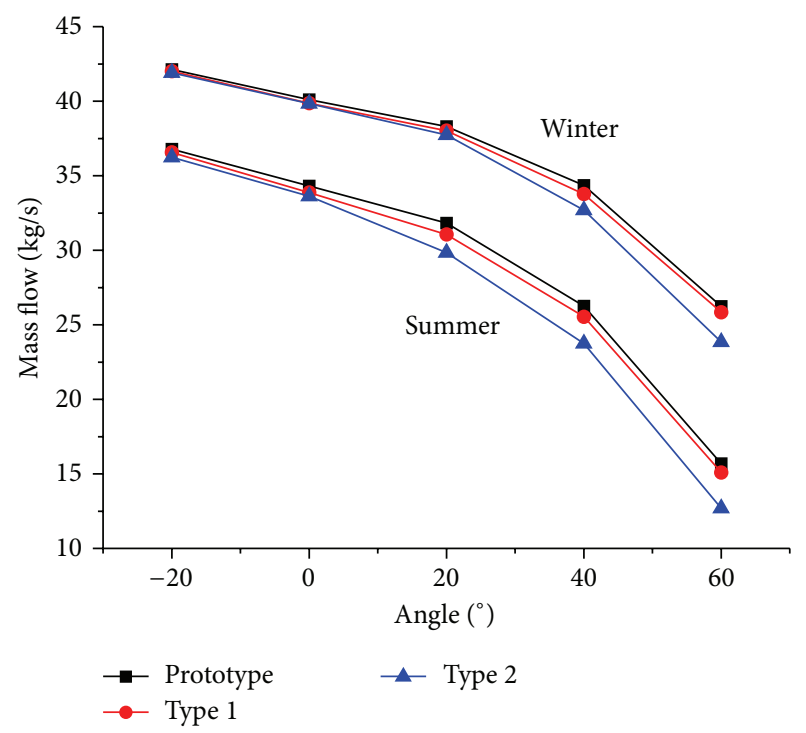

FIGURE 6: The change of mass flow with the setting angles.

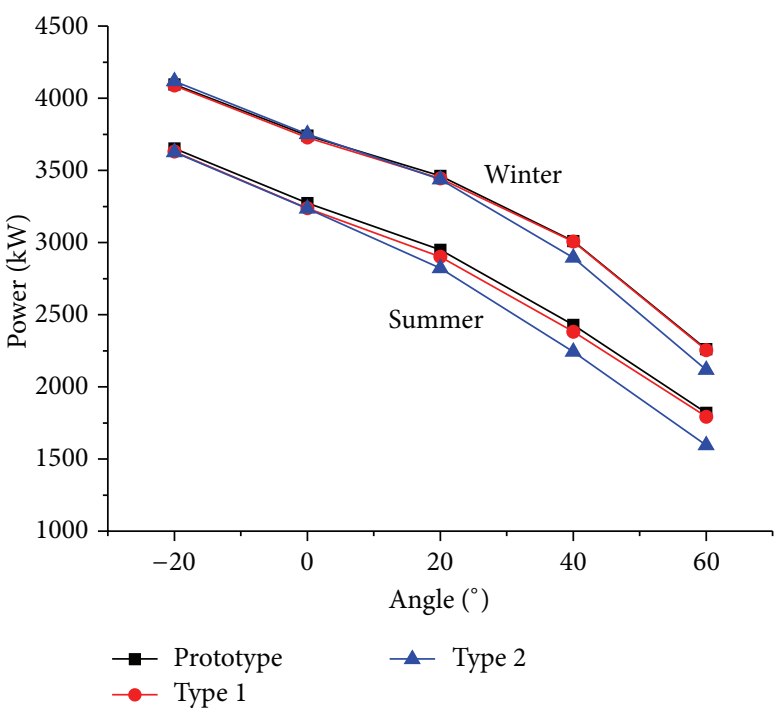

FIGURE 7: The change of power with the setting angles.

of the setting angle. Type 2 makes a lot of power reduction especially in summer, while Type 1 is negligible. As can be seen from Figure 8, the improved structure channel has little effect on the pressure ratio of the compressor. Figure 9 shows the influence of the improved channels on the efficiency of the compressor. The improved structures can significantly improve the efficiency of the compressor, especially in winter, and the higher the setting angle, the higher the efficiency.

The two improved channel structures (Figure 5) wrap the top clearance of the guide vane and thus the leakage of the gas is effectively controlled. As a result, the regulating ability and the performance of the compressor are improved. Although Type 1 can reduce the leakage of gas at the top of the guide vane, there is still a gap left between the vane tip and the box, so that a portion of gas enters the gap, generates flow separation, and increases the flow losses. In the structure 


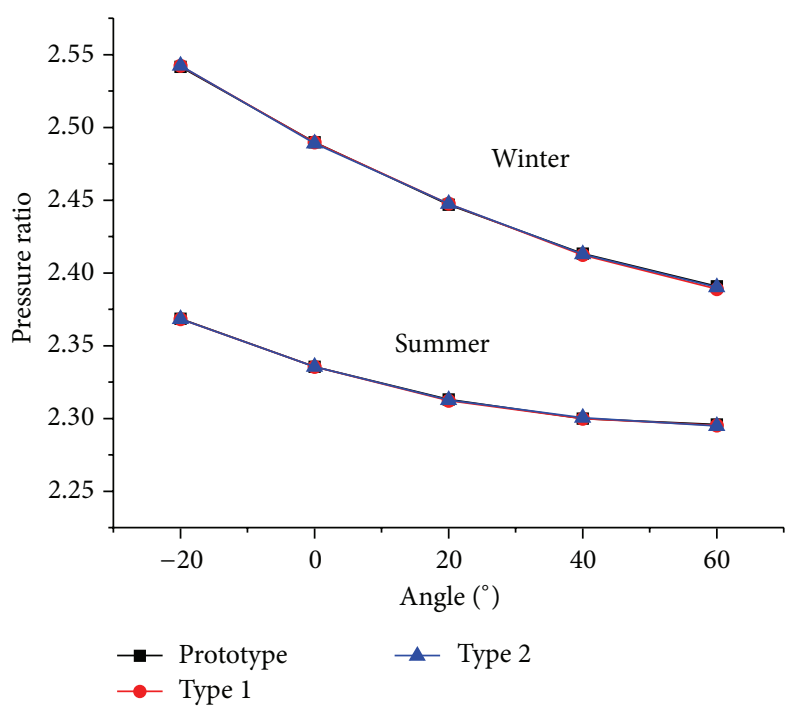

FIGURE 8: The change of pressure ratio with the setting angles.

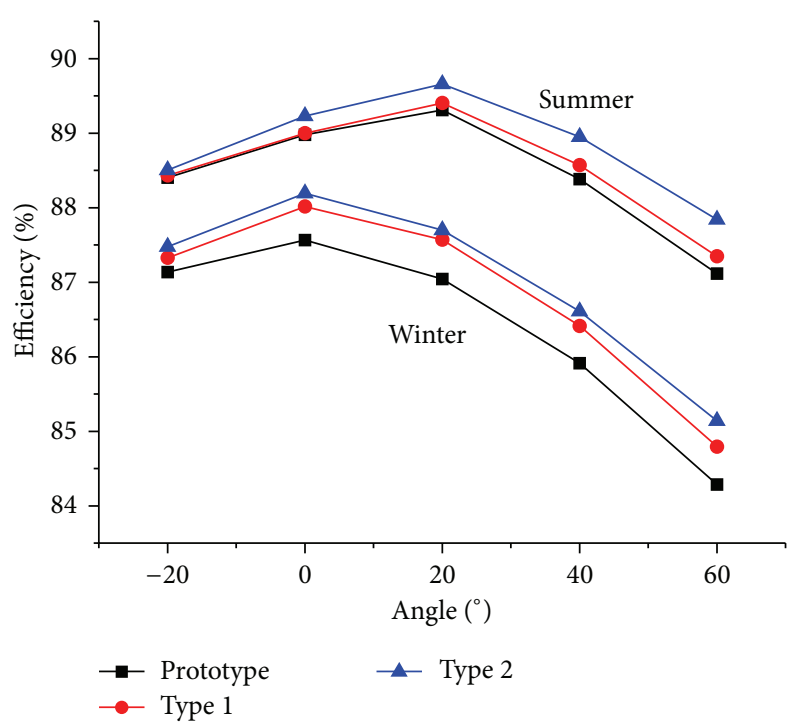

FIgURE 9: The change of efficiency with the setting angles.

of Type 2, the top of the guide vane and the casing wall cooperated with each other so that the secondary flow is limited to the minimum. Obviously, Type 2 is better than Type 1 in that it can not only expand the range of the flow regulation, but also increase the compressor efficiency.

\section{Selection of the Guide Vane Profile}

Considering the vane thickness and camber, ten kinds of NACA profiles were selected for the study. The same numerical calculation method was used for the flow analysis of these blades with Type 2 channel structure, and the flow regulation range chart was drawn.

In order to test the effect of the thickness of the guide vane on the flow regulation, three kinds of straight NACA profile with different thickness were selected, as shown in

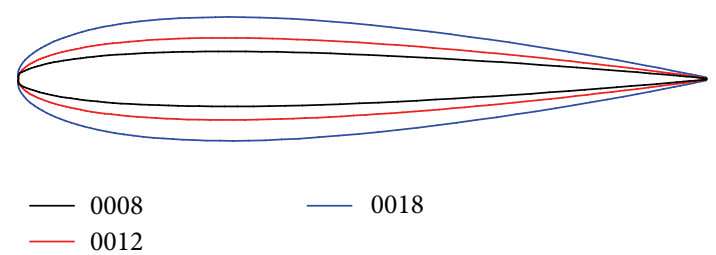

(a) Profiles

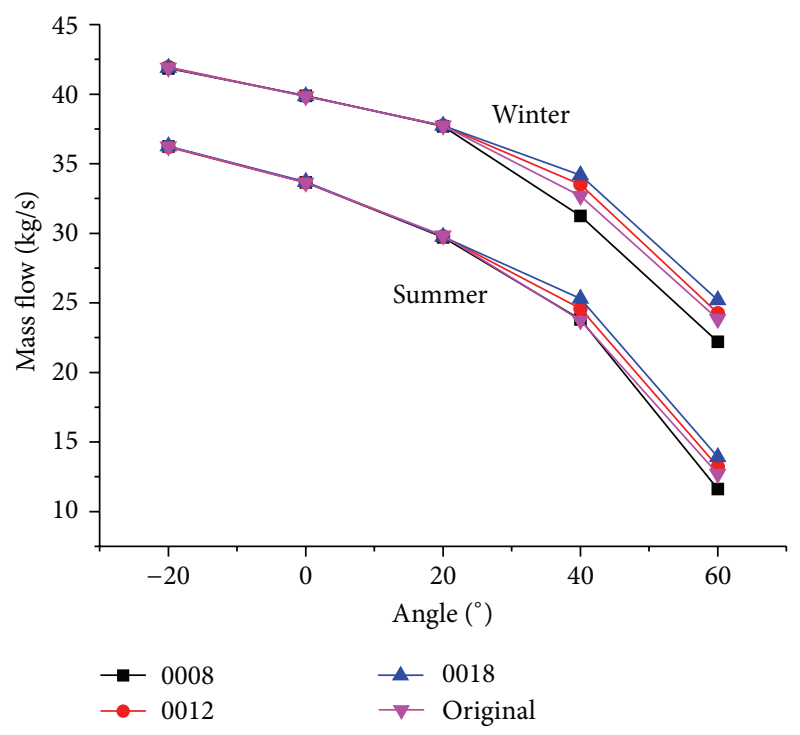

(b) The change of mass flow with the setting angles

FIGURE 10: Straight guide vanes with different thickness.

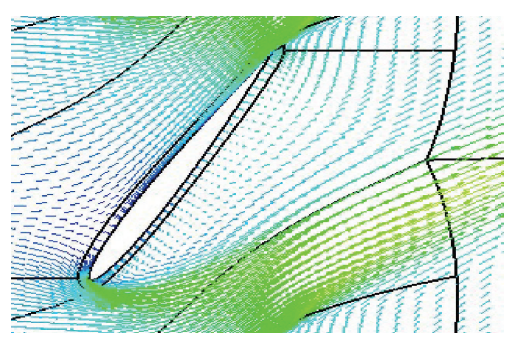

FIGURE 11: Velocity vector distribution of 0008 at $60^{\circ}$ setting angle.

Figure 10(a). Figure 10(b) shows the flow regulation range of these three types of profile, and the comparison with that of the original. As can be seen from the figure, the flow regulation range of the three kinds of profile is the same as that of the original one when the guide vane setting angle is less than 20 degrees. The flow regulation range of the guide vanes is increased with the decrease of the profile thickness when the guide vane setting angle is greater than 20 degrees.

Figures 11 and 12 show the velocity distribution of 0008 and 0018 at $60^{\circ}$ setting angle. From the figures, it can be seen that the speed of gas flow of 0018 is obviously higher than that of 0008. It is because the greater the thickness of the guide vane, the better the performance of the variable angle of attack, so that the flow in the guide vane channel will be more smooth. In contrast, when the gas flows through the thin guide vane, the flow on the suction surface of the guide vane 


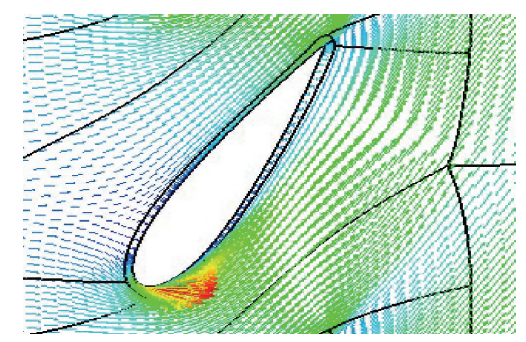

FIGURE 12: Velocity vector distribution of 0018 at $60^{\circ}$ setting angle.

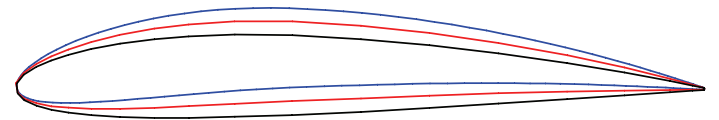

2412
$-\quad 4412$

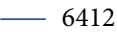

(a) Profiles

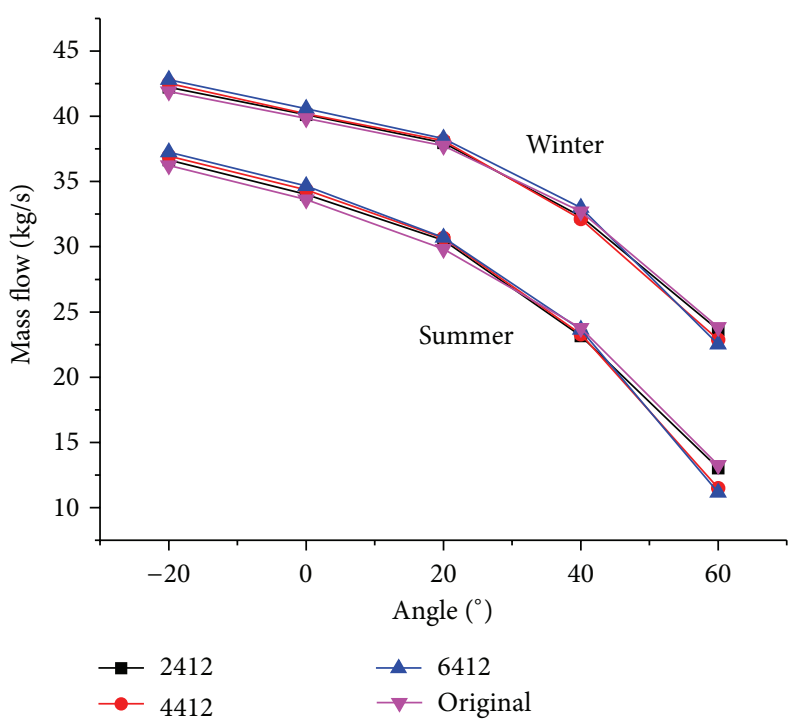

(b) The change of mass flow with the setting angles

FIGURE 13: Different camber guide vanes.

is easy to separate, resulting in a great flow loss. Therefore, reducing the thickness of the guide vane can help us to enlarge the flow regulation range of VIGV, but it may increase the flow loss.

In order to test the effect of the camber of the guide vane on the flow regulation, three kinds of bent NACA profile with different camber were selected, as shown in Figure 13(a). Figure 13(b) shows the flow regulation range of these three types of profile, and the comparison with that of the original. It can be seen from the figure that whether in the large setting angle or in the small setting angle, the flow regulation range of the three kinds of profile is improved to some extent. Among them, the maximum camber 6412 profile flow regulating range is the biggest, and camber minimum 2412 flow regulation scope changes not obviously. Therefore, the

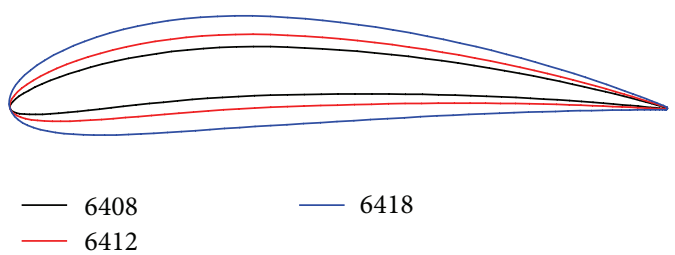

(a) Profiles

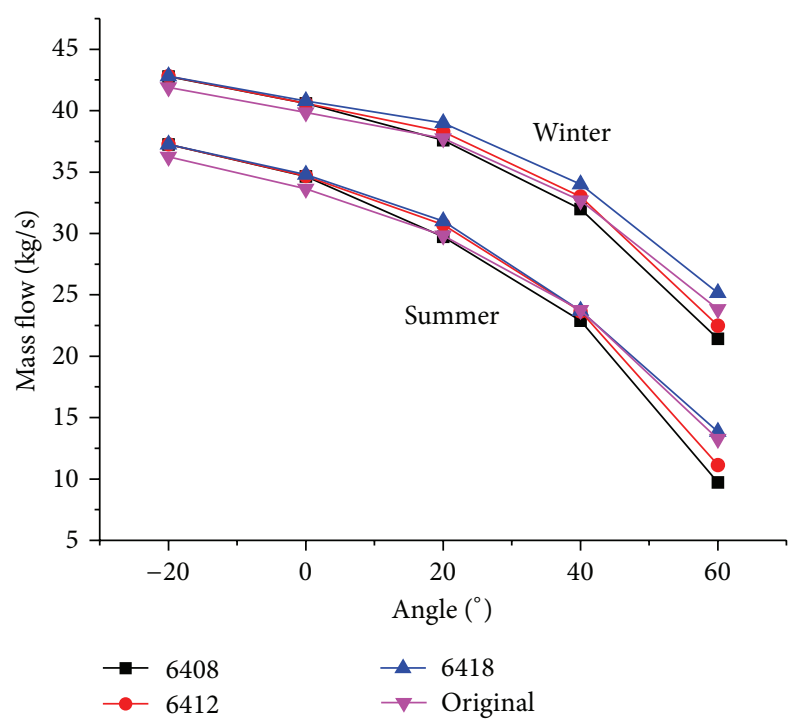

(b) The change of mass flow with the setting angles

FIGURE 14: Camber guide vanes with different thickness.

increase of guide vane camber can help us to expand the range of flow regulation.

In order to test the effect of thickness of the bent guide vane on the flow regulation, based on the 6412 profile, we adjust the thickness of bent guide vane (as shown in Figure 14(a)) and compare the flow regulation range with the original one (as shown in Figure 14(b)). As can be seen from the figure, the same as the case of the straight profile, the greater the thickness of the guide vane, the smaller the flow adjustment range, and the smaller the thickness of the guide vane, the wider the flow adjustment range. The flow regulation range of 6418 is less than the original one. Although 6408 can increase the flow regulation range of the guide vanes, performance calculation indicates that the efficiency of the compressor is greatly decreased at $60^{\circ}$ setting angle, because a strong vortex is generated on the suction surface of the guide vane; the flow separation loss is greatly increased, as shown in Figure 15. Therefore, increasing or decreasing the thickness of 6412 is not conducive to the flow regulation of guide vane.

Based on the 6412 profile, to adjust the maximum camber position of profile (as shown in Figure 16(a)), and to compare the flow regulation range with the original one (as shown in Figure 16(b)), it was found that the curves nearly coincide together for these three profiles and they can improve the flow regulation scope of VIGV. By comparing the three profiles, 6512 has a slight advantage, so the maximum camber location 
TABLE 2: Mass flow, the mass flow rate change ratio, and efficiency at $60^{\circ}$ setting angle.

\begin{tabular}{|c|c|c|c|c|c|c|}
\hline \multirow{2}{*}{ Profile } & \multicolumn{3}{|c|}{ Summer } & \multicolumn{3}{|c|}{ Winter } \\
\hline & Mass flow $(\mathrm{kg} / \mathrm{s})$ & Mass flow change ratio (\%) & Efficiency (\%) & Mass flow $(\mathrm{kg} / \mathrm{s})$ & Mass flow change ratio (\%) & Efficiency (\%) \\
\hline Original & 13.265 & 0 & 87.116 & 23.838 & 0 & 84.285 \\
\hline 0008 & 11.616 & -12.43 & 84.644 & 22.6 & -5.19 & 83.773 \\
\hline 0012 & 12.7 & 4.24 & 87.376 & 24.271 & 1.82 & 84.903 \\
\hline 0018 & 13.92 & 4.94 & 88.074 & 25.2 & 5.71 & 86.000 \\
\hline 2412 & 13.031 & -1.76 & 87.057 & 23.617 & -0.927 & 84.207 \\
\hline 4412 & 11.8 & -11.04 & 86.444 & 22.874 & -4.04 & 83.504 \\
\hline 6412 & 11.49 & -13.38 & 85.763 & 22.4 & -6.03 & 83.084 \\
\hline 6408 & 10.01 & -24.54 & 80.740 & 21.411 & -10.18 & 81.580 \\
\hline 6418 & 13.856 & 4.46 & 87.758 & 25.162 & 5.55 & 84.526 \\
\hline 6312 & 12.11 & -8.71 & 84.607 & 22.52 & -5.53 & 82.207 \\
\hline 6512 & 10.95 & -17.45 & 87.821 & 22.016 & -7.64 & 85.021 \\
\hline
\end{tabular}

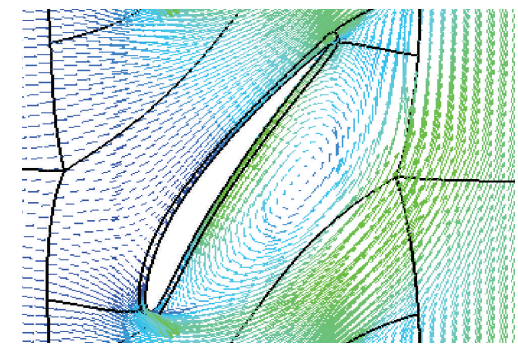

FIGURE 15: Velocity vector distribution of 6408 at $60^{\circ}$ setting angle.

move away from the leading edge can slightly improve the guide vane flow regulating range.

Figures 17 and 18 show the velocity vector distribution of 6512 and original profile at $60^{\circ}$ setting angle. By comparing their flow field distribution, it was found that flow separation occurred at the trailing edge of the suction side of the original guide vane. Instead, 6512 has no obvious flow separation; the flow field distribution is more uniform, reducing the flow loss of the fluid. Therefore, 6512 not only can improve the flow regulation range of the guide vane, but also can reduce the flow loss.

When the guide vane setting angle is 60 degrees, the influence of the mass flow regulation capacity of compressor is the most. Therefore, this paper will further discuss the adjustment ability of the guide vane setting angle at $60^{\circ}$. Table 2 shows the value of the mass flow, the mass flow change ratio, and efficiency of the original profile and 10 kinds of NACA profile at 60 degrees in summer and in winter, respectively. In the table, a negative sign on behalf of the mass flow ratio decreases, and the more it reduces, the greater the flow regulation ability is, and the less the compressed gas wasted. From the numerical value, 0008, 4412, 6412, 6408, and 6512 have better flow regulation capacity; they can expand the flow regulation range of VIGV. Among them, the mass change ratio of 6408 profile is the largest, but the efficiency of the compressor is reduced by using this kind of profile. Overall, the regulation performance of 6512 profile is the best, maximum to expand $17.45 \%$ in summer and $7.64 \%$

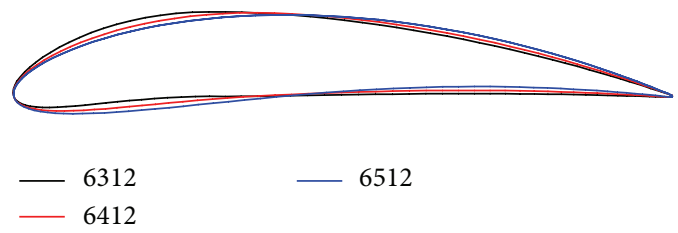

(a) Profiles

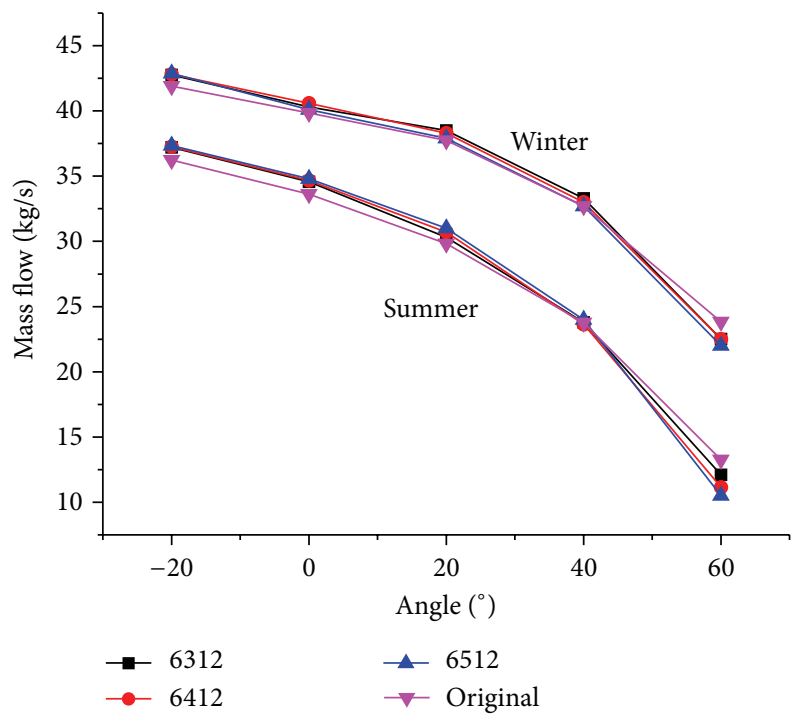

(b) The change of mass flow with the setting angles

FIGURE 16: Guide vanes with different position of camber.

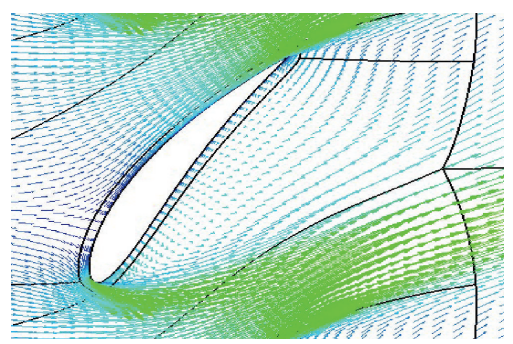

FIGURE 17: Velocity vector distribution of 6512 at $60^{\circ}$ setting angle. 


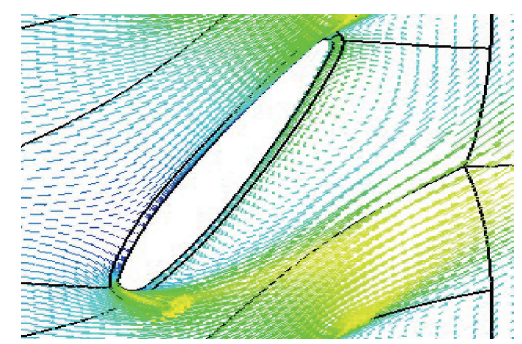

FIGURE 18: Velocity vector distribution of original profile at $60^{\circ}$ setting angle.

in winter, and it improves the efficiency of the compressor slightly.

It can be seen from the table that the change rate of mass flow in summer is higher than that in winter. Although this is the case, the expansion of the flow regulation range of the guide vanes in winter is more significant, because the evacuation of compressed gas is usually higher in winter than that in summer due to the principle of thermal expansion and contraction. Therefore, we are more concerned about the flow regulation capacity of VIGV in winter.

\section{Conclusion}

The two improved channels in this paper can effectively eliminate the influence of the tip clearance on the flow regulation of VIGV. And they can improve the performance of compressor, such as reducing power and increasing efficiency. Type 2 is much better than Type 1; the maximum expansion of mass flow regulation range is $15.19 \%$ in summer and $9.16 \%$ in winter.

Reducing the guide vane thickness, increasing the guide vane camber, and increasing the distance between the maximum camber position and the leading edge of guide vane can help expand the range of mass flow regulation. Considering the performance of compressor, the best guide vane profile is NACA 6512; it can expand the range of mass flow regulation by $17.45 \%$ in summer and $7.64 \%$ in winter.

In conclusion, combined with the structure of Type 2 channel, NACA 6512 as the guide vane profile can expand the mass flow regulation range by $32.64 \%$ in summer and $16.8 \%$ in winter; at the same time the performance of the compressor is slightly increased.

\section{Conflict of Interests}

The authors declare that there is no conflict of interests regarding the publication of this paper.

\section{References}

[1] J.-J. Tan, Y.-J. Mao, D.-T. Qi, R. Wang, and X.-J. Wang, "Research overview on adjustable inlet guide vane for centrifugal compressor," Compressor, Blower \& Fan Technology, vol. 3, pp. 4448, 2006.
[2] A. Mohseni, E. Goldhahn, R. A. Van den Braembussche, and J. R. Seume, "Novel IGV designs for centrifugal compressors and their interaction with the impeller," Journal of Turbomachinery, vol. 134, no. 2, Article ID 021006, 8 pages, 2012.

[3] C.-J. Ji, Z.-Y. Fan, Y. Zhang, and W.-H. Ji, "Study on blade profiles of variable inlet guide vanes of centrifugal compressor," Journal of Thermal Science and Technology, vol. 12, no. 2, pp. 131134, 2013

[4] J. Xiao, C.-G. Gu, C. Gao, and X.-W. Shu, "Numerical analysis of the flow in centrifugal compressor in consideration of inlet prewhirl," Journal of Power Engineering, vol. 28, no. 3, pp. 400-403, 2008.

[5] S. Leichtfuss, C. Biela, H.-P. Schiffer, and F. Heinichen, "Influence of inlet guide vane wakes on the passage flow in a transonic axial compressor," in Proceedings of the ASME Turbo Expo: Turbine Technical Conference and Exposition, Paper no. GT201269485, pp. 2721-2729, Copenhagen, Denmark, June 2012.

[6] Y. N. Chen, D. Hagelstein, I. Kassens, H. Hasemann, U. Haupt, and M. Rautenberg, "Overshoot of the rankine vortex formed in the flow field behind the inlet guide vane of centrifugal compressors," in Proceedings of the ASME International Gas Turbine and Aeroengine Congress and Exhibition, Paper no. 99GT-182, Indianapolis, Ind, USA, June 1999.

[7] D. Händel, R. Niehuis, and U. Rockstroh, "Aerodynamic investigations of a variable inlet guide vane with symmetric profile," in Proceedings of the ASME Turbo Expo: Turbine Technical Conference and Exposition (GT '14), pp. 1-9, Düsseldorf, Germany, June 2014.

[8] R. Guo, L.-P. Jia, X.-L. Fan, Y.-F. Liu, Z. Zhu, and X.-H. He, Series of Tutorials of NUMECA, China Machine Press, Beijing, China, 2013. 


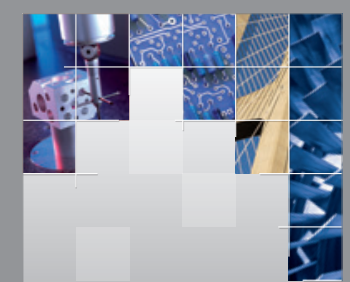

\section{Enfincering}
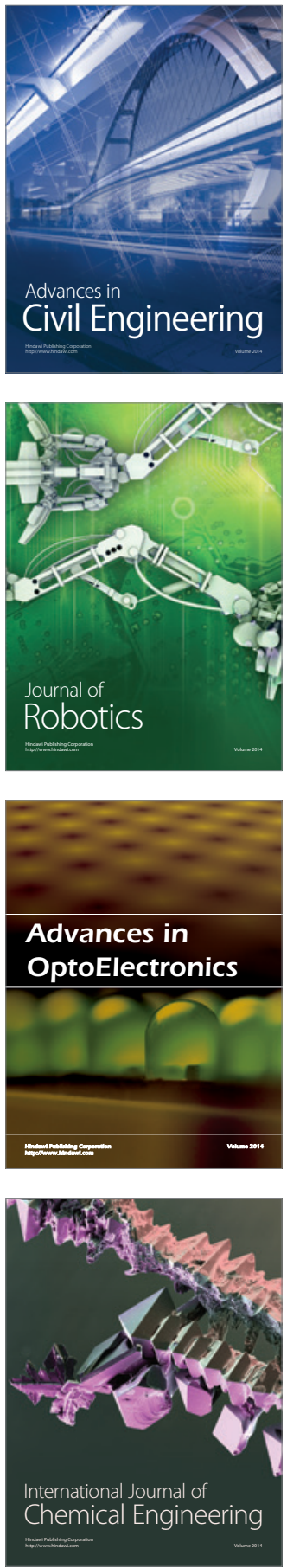

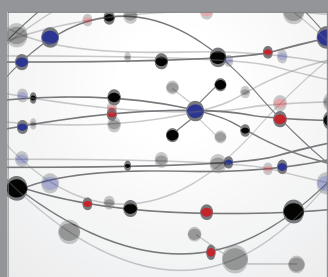

The Scientific World Journal

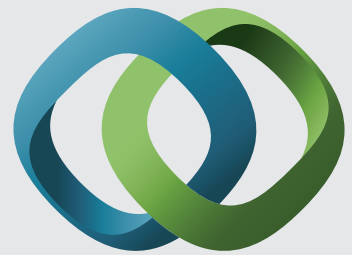

\section{Hindawi}

Submit your manuscripts at

http://www.hindawi.com
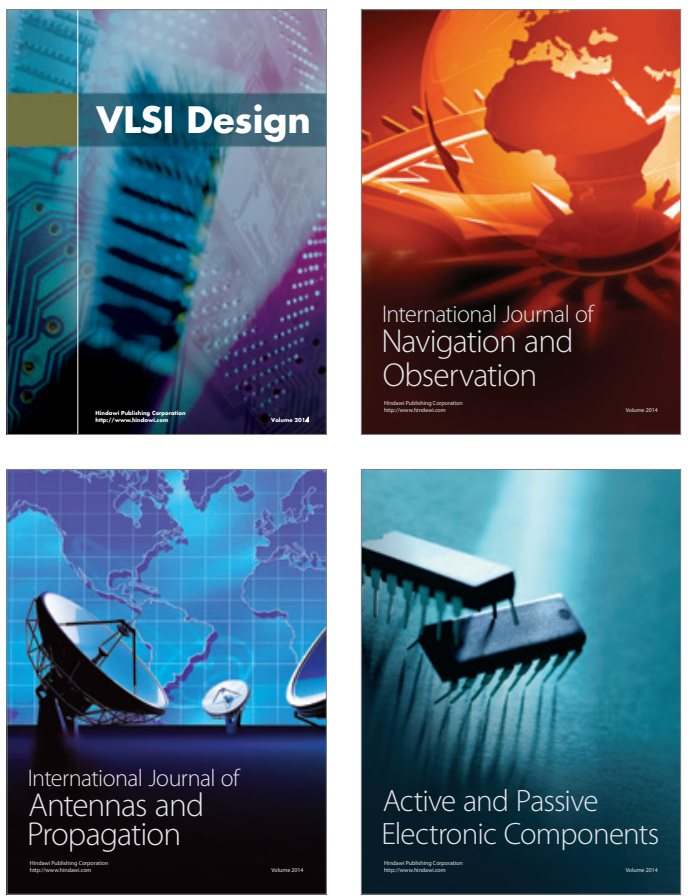
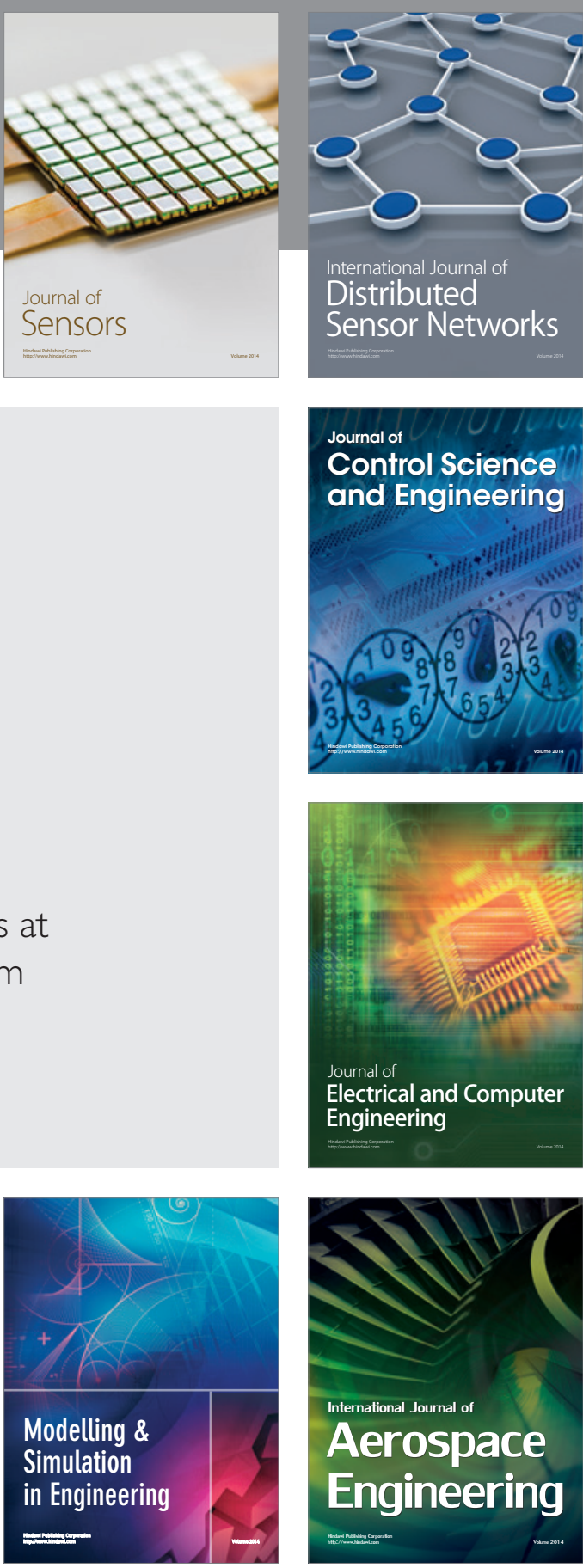

International Journal of

Distributed

Sensor Networks

Journal of

Control Science

and Engineering
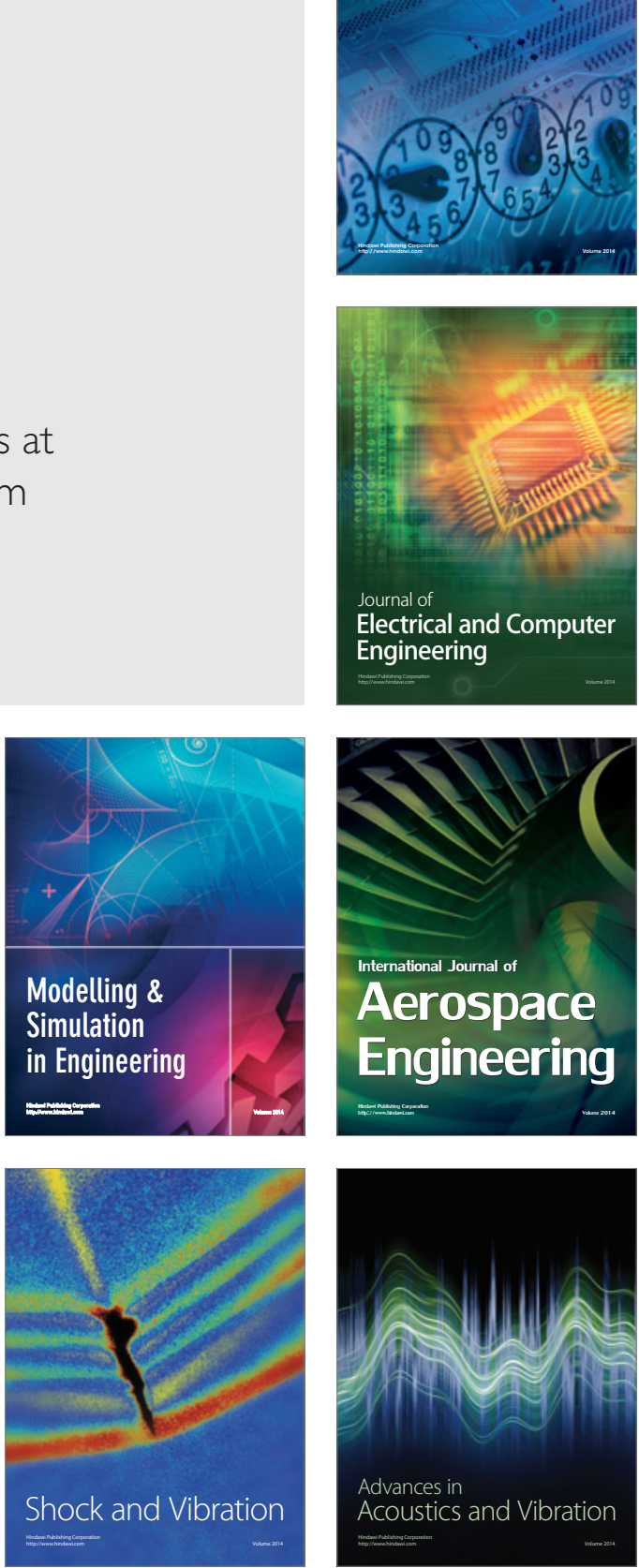